\title{
The FERRUM Project: Experimental transition probabilities of [Fe II] and astrophysical applications
}

\author{
H. Hartman ${ }^{1}$, A. Derkatch ${ }^{2}$, M. P. Donnelly ${ }^{3}$, T. Gull ${ }^{4}$, A. Hibbert ${ }^{3}$, S. Johansson ${ }^{1}$, H. Lundberg ${ }^{5}$, S. Mannervik ${ }^{2}$, \\ L.-O. Norlin ${ }^{6}$, D. Rostohar ${ }^{2}$, P. Royen ${ }^{2}$, and P. Schef ${ }^{2}$ \\ 1 Atomic Astrophysics, Lund Observatory, Lund University, Box 188, 22100 Lund, Sweden \\ 2 Physics Department, Stockholm University, AlbaNova University Center, 10691 Stockholm, Sweden \\ 3 Department of Applied Mathematics and Theoretical Physics, The Queen's University of Belfast, Belfast BT7 1NN, \\ Northern Ireland \\ ${ }^{4}$ NASA/Goddard Space Flight Center, Code 681, Greenbelt, MD 20771, USA \\ 5 Department of Physics, Lund Institute of Technology, Box 118, 22100 Lund, Sweden \\ ${ }^{6}$ Physics Department, Royal Institute of Technology, AlbaNova University Center, 10691 Stockholm, Sweden
}

Received 10 September 2002 / Accepted 25 October 2002

\begin{abstract}
We report on experimental transition probabilities for thirteen forbidden [Fe II] lines originating from three different metastable Fe II levels. Radiative lifetimes have been measured of two metastable states by applying a laser probing technique on a stored ion beam. Branching ratios for the radiative decay channels, i.e. M1 and E2 transitions, are derived from observed intensity ratios of forbidden lines in astrophysical spectra and compared with theoretical data. The lifetimes and branching ratios are combined to derive absolute transition probabilities, $A$-values.

We present the first experimental lifetime values for the two Fe II levels $a^{4} \mathrm{G}_{9 / 2}$ and $b^{2} \mathrm{H}_{11 / 2}$ and $A$-values for 13 forbidden transitions from $a^{6} \mathrm{~S}_{5 / 2}, a^{4} \mathrm{G}_{9 / 2}$ and $b^{4} \mathrm{D}_{7 / 2}$ in the optical region. A discrepancy between the measured and calculated values of the lifetime for the $b^{2} \mathrm{H}_{11 / 2}$ level is discussed in terms of level mixing.

We have used the code CIV 3 to calculate transition probabilities of the $a^{6} \mathrm{D}-a^{6} \mathrm{~S}$ transitions.

We have also studied observational branching ratios for lines from 5 other metastable Fe II levels and compared them to calculated values. A consistency in the deviation between calibrated observational intensity ratios and theoretical branching ratios for lines in a wider wavelength region supports the use of [Fe II] lines for determination of reddening.
\end{abstract}

Key words. atomic data - stars: individual: Eta Carinae - ISM: dust, extinction

\section{Introduction}

By definition metastable states cannot decay through electric dipole (E1) radiation and have therefore long radiative lifetimes $(\approx 1 \mathrm{~s})$. They are, in general, collisionally deexcited in laboratory plasmas. However, in dilute astrophysical plasmas with low probabilities for collisions the metastable states can decay radiatively in magnetic dipole (M1) or electric quadrupole transitions (E2), emitting so called forbidden lines. Such lines can dominate in emission line spectra of low density regions and can be the major cooling agent of dilute plasmas. The use of forbidden lines in the diagnostics of a plasma requires the knowledge of the transition probabilities. These are obtained from theoretical calculations since experimental data require measurements of both the radiative lifetime and the branching fractions. Neither of these measurements is easily performed in the laboratory.

Send offprint requests to: H. Hartman, e-mail: Henrik. Hartman@astro.lu.se
The first identification of forbidden Fe II lines, [Fe II], was done in the pioneering work by Merrill (1928) on the emission line spectrum of $\eta$ Carinae. The identification was stimulated by Bowen's explanation of the nebular lines as M1 transitions between states within the ground configuration of O III (Bowen 1928). The high spatial resolution of the Hubble Space Telescope (HST) made it possible to show that the narrow, strong nebular [Fe II] lines in $\eta$ Car emanate from compact gas ejecta (Davidson et al. 1995), close to the central star. Later HST/STIS spectra (Gull et al. 1999; Gull \& Ishibashi 2001) show the distribution of [Fe II] in the proximity of the star. It is clear that $[\mathrm{Fe} \mathrm{II}]$ provides possibilities to improve the diagnostics of the ejecta of this mysterious star.

The presence of strong [Fe II] lines in the ejecta of $\eta$ Car has initiated the present laboratory study, where we combine measurements of radiative lifetimes of four metastable Fe II levels with theoretical and observational data for the branching fractions in order to get absolute transition probabilities, $A$-values. As is the situation with $A$-values (or $g f$-values) of allowed lines, we will only be able to provide experimental data 
for a few forbidden lines. The main bulk of the data for astrophysical analyses must be supplied by theoretical calculations. It is therefore important to try to get an estimate of the accuracy of the calculated data by experiments. The radiative lifetime of the upper level of a forbidden transition is the atomic parameter that offers a comparison between theory and experiment. We have in our experiments chosen such metastable states of Fe II which are associated with strong nebular [Fe II] lines. One level is known to be affected by level mixing (Johansson 1978) and will provide a critical test of the theoretical calculations.

In the present paper we describe briefly the lifetime measurements in Sect. 2. A more detailed discussion is given in previous papers (Mannervik et al. 1996; Rostohar et al. 2001b). In Sect. 3 we discuss in detail the method of getting branching ratios from astrophysical observations by using HST/STIS spectra of $\eta$ Carinae and its ejecta. New calculations using the CIV3 code on the $a^{6} \mathrm{D}-a^{6} \mathrm{~S}$ transitions are reported in Sect. 4. The lifetimes of four metastable levels of Fe II and transition probabilities for 13 [Fe II] lines are presented in Sect. 5. In Sect. 6 we illustrate the effect of level mixing.

\section{Lifetime measurement of metastable Fe II levels}

The atomic structure of Fe II is complex due to seven valence electrons in one, two or even three open shells. The low complex of even parity configurations $(3 d+4 s)^{7}$ has 62 metastable levels in the energy range $0-4.7 \mathrm{eV}$. All of these levels are easily populated in stellar atmospheres of moderate temperature, which explains the richness in Fe II absorption lines in stellar spectra. The levels are also populated in dilute astrophysical plasmas, where they decay radiatively by forbidden transitions. The potential use of the [Fe II] lines in the diagnostics of astrophysical plasmas is a strong incentive for measuring the radiative lifetime of some metastable states.

The radiative lifetimes of metastable states are typically 6-8 orders of magnitude longer than those of the states decaying by E1 radiation. Consequently, a completely different technique has to be used. Firstly, the $\mathrm{Fe}^{+}$ions have to be stored in a low-density environment during the measurement to avoid collisional quenching. Secondly, the ions have to be stored under controlled conditions for a time comparable to the lifetime. Thirdly, among all the $\mathrm{Fe}^{+}$ions produced and stored, the ones populating the metastable state to be measured have to be unambigously selected and recognized.

A suitable method that meets these criteria has been developed at CRYRING, the storage ring facility at Manne Siegbahn Laboratory (MSL) in Stockholm (Abrahamsson et al. 1993). This method, which is called the laser probing technique (LPT) (Lidberg et al. 1999; Mannervik 2002), has been applied to Fe II (Rostohar et al. 2001b). The $\mathrm{Fe}^{+}$ions were produced from $\mathrm{FeCl}_{2}$ in an ion source, accelerated up to an energy of $40 \mathrm{keV}$ and injected into a storage ring. The long radiative lifetimes of the metastable levels require that the ring is evacuated to a pressure of $10^{-11}$ torr to avoid collisional quenching with the rest gas. The particular state to be measured is selected by irradiating the iron beam with monochromatic light from a cw laser tuned at a frequency matching an allowed transition to a higher state. The metastable state is thus depleted and excited to a state

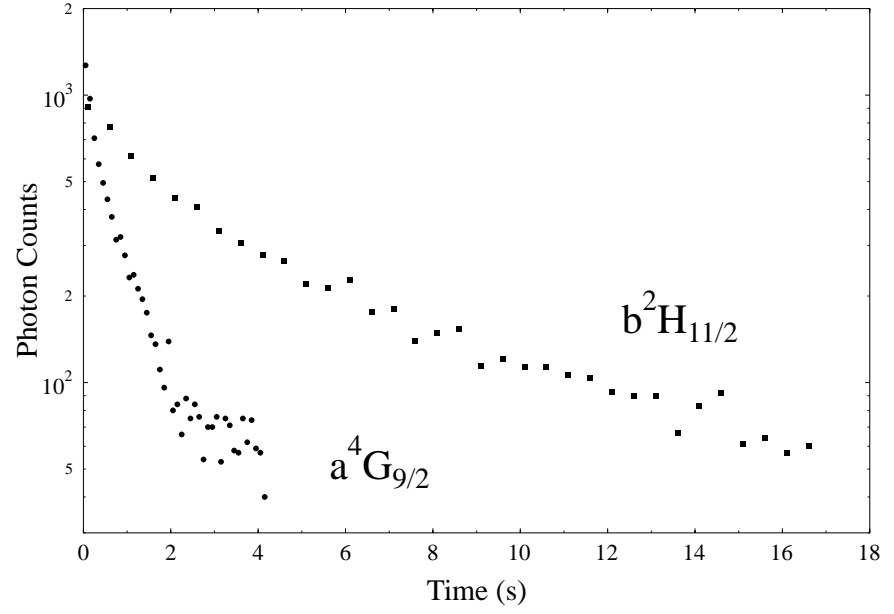

Fig. 1. Typical lifetime curves (after corrections) for the levels $a^{4} \mathrm{G}_{9 / 2}$ and $b^{2} \mathrm{H}_{11 / 2}$.

of opposite parity. This short-lived state decays in a fast transition and the corresponding fluorescence signal is detected. The laser probing pulse is systematically applied at different time delays after the injection of the iron beam into the storage ring. Thus, the number of fluorescent photons is monitored as a function of time and a lifetime curve is obtained. Certain correction and normalization procedures have to be applied, and all such details are given in previous papers (e.g. Rostohar et al. 2001a; Rostohar et al. 2001b). Examples of lifetime curves are given in Fig. 1.

\section{Measurements of branching ratios using astrophysical spectra}

The relevant parameter in astrophysical analyses is the transition probability $\left(A_{i k}\right)$ which is related to the measurable quantities, branching fraction $\left(B F_{i k}\right)$ and radiative lifetime $\left(\tau_{i}\right)$ by

$A_{i k}=B F_{i k} / \tau_{i}$

where

$B F_{i k}=A_{i k} / \sum_{k} A_{i k}$ and $\tau_{i}=\left(\sum_{k} A_{i k}\right)^{-1}$.

The upper and lower states are denoted by $i$ and $k$, respectively. $B F_{i k}$ is obtained by measuring the intensity (in photons per second) of the emission line $\lambda_{i k}$ relative to the total intensity of all emission lines from level $i$.

Lifetimes of metastable states are typically 6-8 orders of magnitude longer than of ordinary excited levels, and the transition probabilities of the forbidden lines are accordingly very small. To avoid collisional quenching of the metastable states a very low electron density (typically $10^{5} \mathrm{~cm}^{-3}$ ) is required in a plasma emitting observable forbidden lines. In general, this also means that a low concentration of ions is required throughout an extended source. Thus, the combination of low density and low transition probability makes dilute, extended astrophysical plasmas the best environment to produce forbidden lines of significant flux, where the low volume density is 
compensated by a large column density. In rare cases, forbidden lines have been observed in laboratory sources allowing accurate wavelength measurements (Eriksson 1965).

We have considered two ways to get the $B F$ 's and to derive the $A$-values:

1) Measure the relative intensities of the relevant [Fe II] lines in astrophysical spectra;

2) Use theoretical calculations of forbidden transitions in Fe II. We have finally chosen a combination of the two, but before describing the procedure, we comment on the two different methods. The main argument against using astrophysical spectra is of course that we want to derive the atomic parameter values in an independent way and use them in the astrophysical analysis. A practical problem with the astrophysical spectra is that the intensities are affected by interstellar extinction and may not provide the "true" branching fractions. Also, a nebular emission line may be blended with other lines from the same or another ion. This makes the intensity measurements less reliable, even if the blending component is known. The general problem with method 2) is the difficulty in estimating reliable uncertainties of calculated values.

We will adopt the following approach: We construct a reddening curve for one particular spectrum of ejecta near $\eta$ Car by using theoretical BF's for a number of [Fe II] lines. We correct the observed intensities of the lines under investigation and use them to derive branching fractions.

\subsection{Construction of a reddening curve for $\eta$ Carinae}

We have measured the observed intensities (in photons per s) of 14 [Fe II] lines in the HST/STIS spectrum of two compact gaseous condensations (called the Weigelt blobs B and D, Weigelt \& Ebersberger 1986) of $\eta$ Carinae and grouped the lines according to their upper energy level. These lines have been observed at three occasions: March 1998, February 1999 and March 2000. The line ratios from the different observations show consistent results. We used the March 1998 data for the analysis since the lines of interest are less affected by blending of other lines. The observed intensity ratio between pairs of lines from a common upper level, i.e. the uncalibrated branching ratio, is compared to the theoretical branching ratio. For the latter we have used the calculations by Quinet et al. (1996) as they give a good agreement with the experimental lifetimes (Rostohar et al. 2001b). By using a standard reddening curve (Osterbrock 1989) with a wavelength dependent exponential degrading of the intensity, we can derive the amount of reddening for each line pair. If the exponential function is a good approximation the different line pairs would give the same value of the exponential constant $C$. By averaging over 11 pairs of [Fe II] lines, we derive a value of $C=1.6 \pm 0.4$. The variation in $C$ is caused by line blending, by uncertainties in the flux measurements and in the calculations of transition probabilities. Some of the linepairs used may have larger errors according to a note in proof on the paper by Quinet et al. (1996). This has been taken into consideration when deriving the uncertainties of the $A$-values. However, there is some evidence

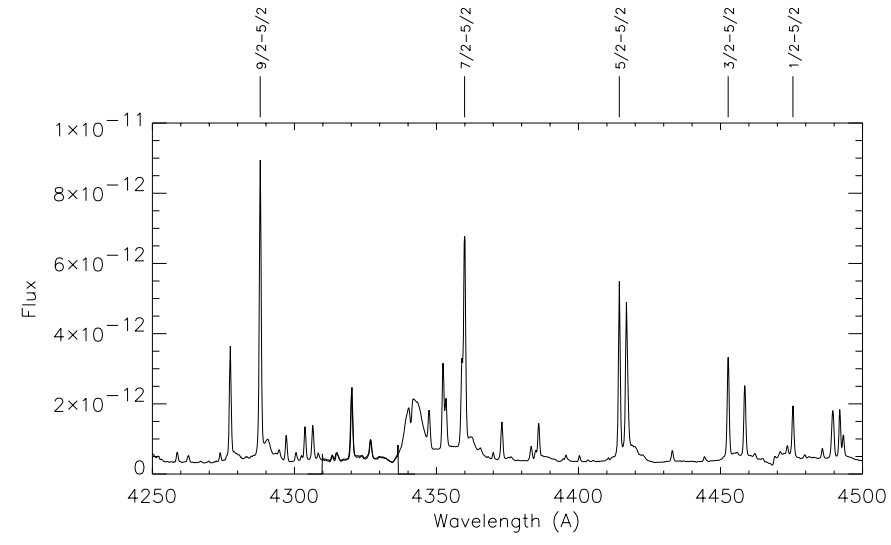

Fig. 2. HST/STIS spectrum of the blob spectrum of Eta Carinae showing the strong lines $a^{6} \mathrm{D}-a^{6} \mathrm{~S}$.

that nebular reddening in the Homonculus may not follow the standard interstellar reddening curve.

\subsection{Calibrated branching ratios}

We use the reddening curve to derive calibrated intensities and branching fractions for the forbidden lines associated with the four metastable Fe II levels, for which we have experimental lifetimes. For one of the levels, $a^{6} \mathrm{~S}$, the lines fall in a very narrow region (4280-4480 $\AA$, see Fig. 2) so we can, to a first approximation, neglect the effect of reddening. We compare the $B F$ 's derived from the nebular intensities with calculated $B F$ 's (Quinet et al. 1996) in Fig. 3, and find an extremely good agreement.

We have repeated the same procedure for two other levels, $b^{2} \mathrm{H}_{11 / 2}$ and $a^{4} \mathrm{G}_{9 / 2}$, which also have their main branches within 100 and $200 \AA$, respectively. The agreement is reasonable good, as shown in Fig. 3 even if one line from $a^{4} \mathrm{G}_{9 / 2}$ is blended. The line at $\lambda 4177$ in the $\eta$ Car spectrum is a blend of the two Fe II transitions $a^{4} \mathrm{~F}_{9 / 2}-a^{4} \mathrm{G}_{9 / 2}$ and $a^{2} \mathrm{D}_{5 / 2}-z^{4} \mathrm{D}_{7 / 2}$. This affects the branching fractions for all lines from the $a^{4} \mathrm{G}_{9 / 2}$ level. To estimate the contribution of the allowed transition to the observed nebular feature, we have measured the flux from other nebular lines from the same upper level, $z^{4} \mathrm{D}_{7 / 2}$. Based on the calculations by Raassen \& Uylings (2000) and on solar spectrum observations (Thevenin 1989), we estimate the contribution from the $a^{2} \mathrm{D}_{5 / 2}-z^{4} \mathrm{D}_{7 / 2}$ line to be $\sim 20 \%$ of the observed emission feature in $\eta$ Carinae.

Based on this good agreement we can now, in principle, combine the theoretical BF's with measured lifetimes for these three levels and derive absolute transition probabilities (see results in Sect. 4).

The branches from the fourth level, $b^{4} \mathrm{D}_{7 / 2}$, are spread over a large wavelength interval, $3100-5600 \AA$, and the effect of reddening is not negligible. In Fig. 4 we show the transition probabilities derived from the stellar intensities, which are corrected for reddening at three different values of $C$ located around the adopted value of 1.6. Assuming that the standard formula for interstellar reddening is a good approximation we see that the error introduced by the uncertainty in the $C$ value is rather small. A change in $C$ of 0.5 introduces a change in the $\mathrm{BF}$ 

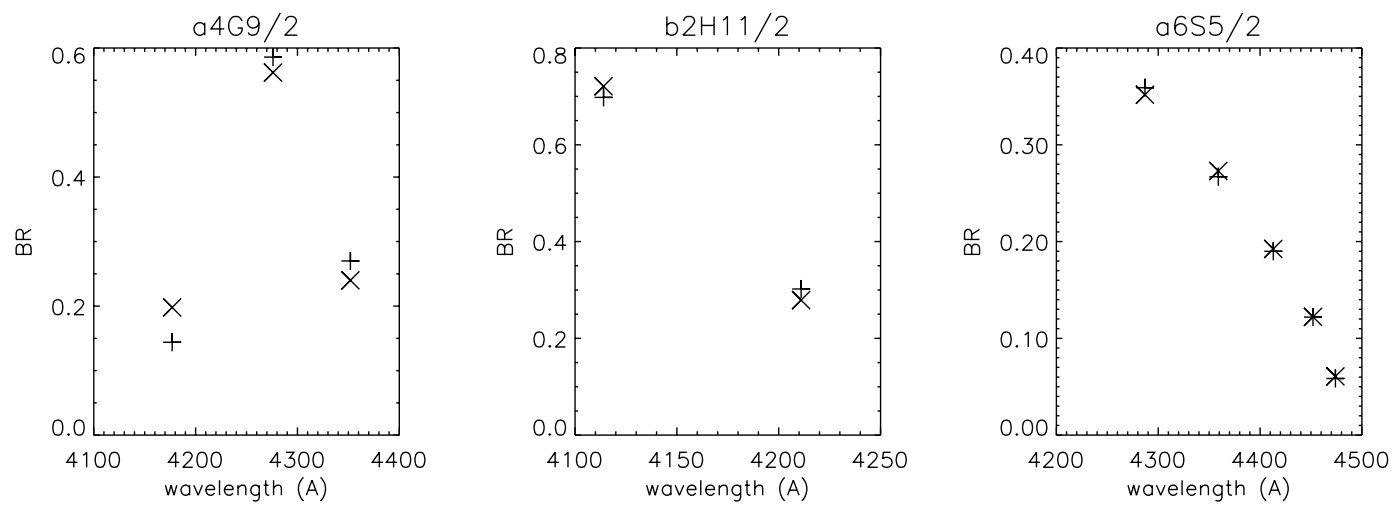

Fig. 3. Branching ratios for the metastable levels $a^{4} \mathrm{G}_{9 / 2}, b^{2} \mathrm{H}_{11 / 2}$ and $a^{6} \mathrm{~S}_{5 / 2}$. Crosses $(\times)$ mark the astrophysical values corrected for reddening and the (+) mark the theoretical values (Quinet et al. 1996).

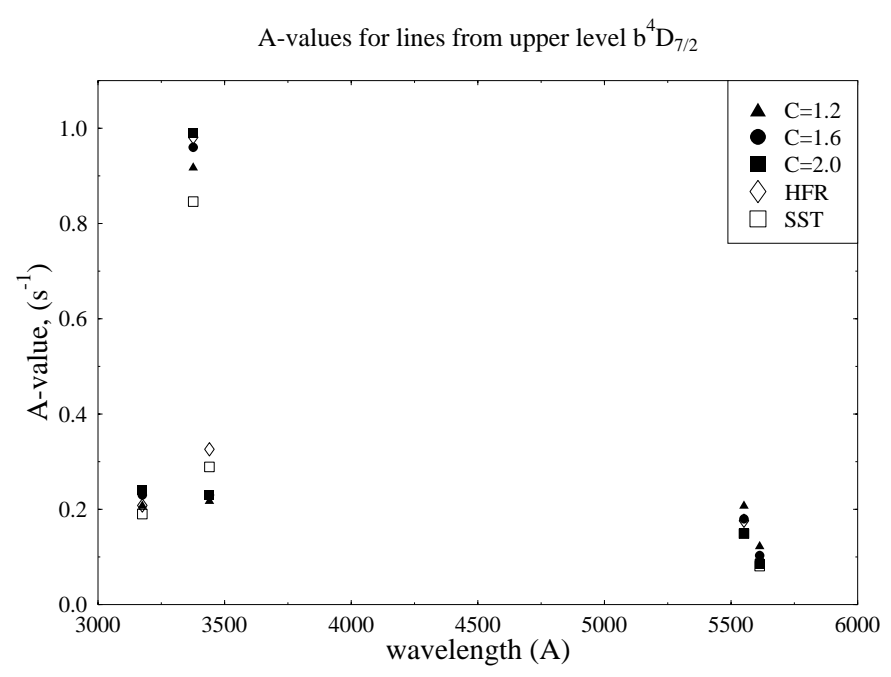

Fig. 4. $A$-values for transitions from the upper level $b^{4} \mathrm{D}_{7 / 2}$ for different values of the reddening constant $C$. The theoretical values (HFR and SST) are from Quinet et al. (1996).

Table 1. Optimisation processes for the radial functions.

\begin{tabular}{clc}
\hline \hline Orbital & Configurations included & Symmetry \\
\hline $4 \mathrm{p}$ & $3 \mathrm{~d}^{5} 4 \mathrm{~s} 4 \mathrm{p}$ & $y^{6} \mathrm{P}^{\mathrm{o}}$ \\
$5 \mathrm{p}$ & $3 \mathrm{~d}^{6} 4 \mathrm{p}, 3 \mathrm{~d}^{6} 5 \mathrm{p}$ & $z^{6} \mathrm{P}^{\mathrm{o}}$ \\
$6 \mathrm{p}$ & $3 \mathrm{~d}^{6} n \mathrm{p} ; n=4,5,6$ & $z^{6} \mathrm{D}^{\circ}$ \\
$7 \mathrm{p}$ & $3 \mathrm{~d}^{6} n \mathrm{p} ; n=4,5,6,7$ & $z^{6} \mathrm{~F}^{\mathrm{o}}$ \\
$5 \mathrm{~s}$ & $3 \mathrm{~d}^{5} 4 \mathrm{~s} 4 \mathrm{p}, 3 \mathrm{~d}^{5} 5 \mathrm{~s} 4 \mathrm{p}$ & $y^{6} \mathrm{P}^{\mathrm{o}}$ \\
$6 \mathrm{~s}$ & $3 \mathrm{~d}^{5} m \mathrm{~s} n \mathrm{p} ; m, n=4,5,6$ & $y^{6} \mathrm{P}^{\mathrm{o}}$ \\
$4 \mathrm{~d}$ & $3 \mathrm{~d}^{6} 4 \mathrm{p}, 3 \mathrm{~d}^{5} 4 \mathrm{~s} 4 \mathrm{p}, 3 \mathrm{~d}^{4} 4 \mathrm{~s} 4 \mathrm{p} 4 \mathrm{~d}$ & $y^{6} \mathrm{P}^{\mathrm{o}}$ \\
$5 \mathrm{~d}$ & $3 \mathrm{~d}^{6} 4 \mathrm{p}, 3 \mathrm{~d}^{5} 4 \mathrm{~s} 4 \mathrm{p}, 3 \mathrm{~d}^{5} 4 \mathrm{p} 4 \mathrm{~d}, 3 \mathrm{~d}^{5} 4 \mathrm{p} 5 \mathrm{~d}$ & $z^{6} \mathrm{P}^{\mathrm{o}}$ \\
$4 \mathrm{f}$ & $3 \mathrm{~d}^{6} 4 \mathrm{~s}, 3 \mathrm{~d}^{5} 4 \mathrm{p} 4 \mathrm{f}$ & $a^{6} \mathrm{D}$ \\
\hline
\end{tabular}

of $\sim 10 \%$ for the UV lines around $3400 \AA$ and $\sim 20 \%$ for the lines at $5500 \AA$.

\section{Calculations of transitions probabilities}

The present work uses CI wave functions of the form

$\Psi(J)=\sum_{i=1}^{M} a_{i} \Phi_{i}\left(\alpha_{i} L_{i} S_{i} J\right)$ generated by the code CIV3 (Hibbert 1975; Hibbert et al. 1991). For a specific choice of configuration state functions (CSFs) $\left\{\Phi_{i}\right\}$, where the angular momentum coupling scheme is defined by $\left\{\alpha_{i}\right\}$, the variationally optimal expansion coefficients $\left\{a_{i}\right\}$ are the eigenvector components of the diagonalized Hamiltonian whose typical element is $H_{i j}=<\Phi_{i}|H| \Phi_{j}>$. The corresponding eigenvalue is an upper bound to the energy of the particular state. In particular, if the eigenvalues $\left\{E_{i}\right\}$ are ordered so that $E_{1}<E_{2}<\cdots$, then

$E_{i} \geq E_{i}^{\text {exact }}$

is a consequence of the Hylleraas-Undheim theorem.

The Hamiltonian used to determine the final wave functions consists of the non-relativistic Schrödinger Hamiltonian along with the following relativistic operators associated with the Breit-Pauli approximation: mass-correction, Darwin, spinspin, spin-other-orbit and spin-orbit terms. However, the spinspin-contact and the orbit-orbit terms, which do not contribute directly to fine-structure separations, are not included in the calculations.

The CSFs of all states are constructed from a common set of one-electron orbitals of the form

$\frac{1}{r} P_{n l}(r) Y_{l}^{m_{l}}(\theta, \phi) \chi_{m_{s}}(\sigma)$

where the radial functions in Eq. (5) are expressed in analytic form as a linear combination of normalized Slater-type orbitals (STOs):

$P_{n l}(r)=\sum_{j=1}^{k} c_{j n l} \chi_{j n l}(r)$

and where the STOs take the form

$\chi_{j n l}(r)=\left[\frac{\left(2 \zeta_{j n l}\right)^{2 I_{j n l}+1}}{\left(2 I_{j n l}\right) !}\right]^{1 / 2} r^{I_{j n l}} \exp \left(-\zeta_{j n l} r\right)$.

Equations (4) constitute a set of variational principles allowing the optimisation of the radial function parameters on one or more of the energy eigenvalues of the Hamiltonian matrix. The integers $\left\{I_{j n l}\right\}$ are kept fixed but the exponents $\left\{\zeta_{j n l}\right\}$ and the coefficients $\left\{c_{j n l}\right\}$ in Eq. (7) may be treated as variational 
Table 2. Optimised radial function parameters for Fe II.

\begin{tabular}{|c|c|c|c|c|c|c|c|c|c|c|c|}
\hline$n l$ & $c_{j n l}$ & $I_{j n l}$ & $\zeta_{j n l}$ & $n l$ & $c_{j n l}$ & $I_{j n l}$ & $\zeta_{j n l}$ & $n l$ & $c_{j n l}$ & $I_{j n l}$ & $\zeta_{j n l}$ \\
\hline \multirow[t]{6}{*}{$5 s$} & 0.07552 & 1 & 19.02548 & $4 d$ & 0.63694 & 3 & 5.15025 & $7 p$ & 14.57355 & 2 & 0.91682 \\
\hline & -0.27401 & 2 & 9.35791 & & -0.92313 & 4 & 1.90298 & & -35.18975 & 3 & 1.57708 \\
\hline & 0.57776 & 3 & 5.07754 & & & & & & 18.84919 & 4 & 1.93717 \\
\hline & -1.03497 & 4 & 2.43824 & $5 \mathrm{~d}$ & 0.44144 & 3 & 4.73722 & & 166.94166 & 5 & 1.93650 \\
\hline & 0.97510 & 5 & 1.29982 & & -0.77547 & 4 & 2.61070 & & -366.00070 & 6 & 2.15513 \\
\hline & & & & & 1.04299 & 5 & 1.28493 & & 200.95182 & 7 & 2.40962 \\
\hline \multirow[t]{7}{*}{$6 s$} & 0.06441 & 1 & 18.38583 & & & & & & & & \\
\hline & -0.16284 & 2 & 11.31165 & $4 p$ & 5.70277 & 2 & 1.44819 & $4 \mathrm{f}$ & 1.00000 & 4 & 2.44380 \\
\hline & 4.62987 & 3 & 2.03315 & & -1.88413 & 3 & 4.04161 & & & & \\
\hline & -10.89075 & 4 & 2.13989 & & -3.85906 & 4 & 2.46303 & & & & \\
\hline & 7.93749 & 5 & 2.07416 & & & & & & & & \\
\hline & -1.98787 & 6 & 1.49966 & $5 p$ & 0.67165 & 2 & 2.83628 & & & & \\
\hline & & & & & -0.78204 & 3 & 4.21964 & & & & \\
\hline \multirow[t]{8}{*}{$7 \mathrm{~s}$} & 0.97432 & 1 & 0.30972 & & 5.72815 & 4 & 0.85910 & & & & \\
\hline & -1.45676 & 2 & 1.62185 & & -6.48385 & 5 & 1.00256 & & & & \\
\hline & 11.78939 & 3 & 1.45012 & & & & & & & & \\
\hline & -28.95904 & 4 & 1.58546 & $6 \mathrm{p}$ & 1.19932 & 2 & 10.90063 & & & & \\
\hline & 31.20386 & 5 & 1.64232 & & -1.37372 & 3 & 10.06883 & & & & \\
\hline & -15.20386 & 6 & 1.64979 & & 7.29654 & 4 & 1.35418 & & & & \\
\hline & 1.40099 & 7 & 1.11660 & & -16.45629 & 5 & 1.35122 & & & & \\
\hline & & & & & 9.70525 & 6 & 1.40443 & & & & \\
\hline
\end{tabular}

Table 3. Ab initio and fine-tuned results for calculation including all one- and two-electron changes from $\left(3 d^{6} 4 s, 3 d^{5} 4 s 4 d\right){ }^{6} D$ and $\left(3 d^{5} 4 s^{2}\right.$, $\left.3 d^{5} 4 s 5 s, 3 d^{5} 4 p^{2}, 3 d^{5} 4 s 4 d\right){ }^{6} S$.

\begin{tabular}{cccccccc}
\hline \hline \multicolumn{2}{c}{ Transition } & \multicolumn{3}{c}{ Ab initio results } & \multicolumn{3}{c}{ Fine-tuned results } \\
Upper level & Lower level & $\Delta E\left(\mathrm{~cm}^{-1}\right)$ & $f_{l}$ & $A_{l}\left(\mathrm{~s}^{-1}\right)$ & $\Delta E\left(\mathrm{~cm}^{-1}\right)$ & $f_{l}$ & $A_{l}\left(\mathrm{~s}^{-1}\right)$ \\
\hline$a^{6} \mathrm{~S}_{5 / 2}$ & $a^{6} \mathrm{D}_{9 / 2}$ & 22020.67 & 0.0846 & 0.9777 & 23317.40 & 0.0894 & 1.6247 \\
& $a^{6} \mathrm{D}_{7 / 2}$ & 21643.88 & 0.0837 & 0.9030 & 22932.64 & 0.0886 & 1.2042 \\
& $a^{6} \mathrm{D}_{5 / 2}$ & 21355.16 & 0.0830 & 0.6367 & 22649.56 & 0.0879 & 0.8533 \\
& $a^{6} \mathrm{D}_{3 / 2}$ & 21151.27 & 0.0825 & 0.4061 & 22455.02 & 0.0875 & 0.5469 \\
& $a^{6} \mathrm{D}_{1 / 2}$ & 21029.86 & 0.0823 & 0.1977 & 22340.36 & 0.0873 & 0.2671 \\
\hline
\end{tabular}

parameters to be optimised subject to the orthonormality conditions:

$\int_{0}^{\infty} P_{n l}(r) P_{n^{\prime} l}(r) \mathrm{d} r=\delta_{n n^{\prime}} ; \quad l<n^{\prime} \leq n$.

The optimisation of the radial functions on, for example, the energy of just one state would lead to a serious imbalance in the accuracy to which the full set of states under consideration would be represented. Hence different orbitals are optimised on different eigenvalues, so that all the states are of a comparable level of accuracy. In the present work, the $1 \mathrm{~s}, 2 \mathrm{~s}$, 2 p, 3s, 3p, 3d, 4s functions were taken from the ground state Hartree-Fock functions given by Clementi \& Roetti (1974): hence they are all optimised on the $a^{6} \mathrm{D}$ state. In the present calculations we used the radial functions previously optimised in our calculations of the $a^{6} \mathrm{D}-z^{6} \mathrm{P}^{0}$ transitions (Donnelly $\&$ Hibbert 2001). The additional orbitals consist of $5 \mathrm{~s}, 6 \mathrm{~s}$, $4 \mathrm{p}, 5 \mathrm{p}, 6 \mathrm{p}, 4 \mathrm{~d}, 5 \mathrm{~d}, 4 \mathrm{f}$. The method of optimisation (involving only the non-relativistic Schrödinger Hamiltonian) is displayed in Table 1 and the radial function parameters are given in Table 2. We give in Table 3 both our ab initio results of oscillator strengths and the corresponding values obtained after the "fine-tuning" of the calculated energy levels. This process
(Brage \& Hibbert 1989) makes small adjustments to the diagonal elements of the Hamiltonian matrix so as to bring the calculated eigenvalue differences into agreement with the corresponding experimental energy differences. This process has proved effective in improving the accuracy of calculated oscillator strengths (Hibbert 1996). The adopted $A$-values are inserted in Table 5 and compared with the new experimental and previously calculated values.

\section{Results and discussion}

Radiative lifetimes of metastable states in Fe II were first measured for two levels, $a^{6} \mathrm{~S}_{5 / 2}$ and $b^{4} \mathrm{D}_{7 / 2}$, and they were reported in Rostohar et al. (2001b). In the present paper we report on the lifetime measurement of two more levels, $a^{4} \mathrm{G}_{9 / 2}$ and $b^{2} \mathrm{H}_{11 / 2}$. All values are presented in Table 4, where we also compare with theoretical calculations by Garstang (1962), Nussbaumer et al. (1981), Quinet et al. (1996) and our new calculations. The theoretical lifetimes are obtained by taking the inverse sum of all $A$-values (see Eq. (1)), which is the parameter calculated by the codes. Nussbaumer et al. (1981) made calculations only for the system of sextet levels, and the good agreement between their value for the $a^{6} \mathrm{~S}$ term and our experimental value implies 
Table 4. Lifetimes (in s) of metastable Fe II levels.

\begin{tabular}{|c|c|c|c|c|c|}
\hline & & $\overline{b^{4} \mathrm{D}_{7 / 2}}$ & $a^{6} \mathrm{~S}_{5 / 2}$ & $\overline{a^{4} \mathrm{G}_{9 / 2}}$ & $\overline{b^{2} \mathrm{H}_{11 / 2}}$ \\
\hline Garstang (1962) & & 0.618 & 0.326 & 0.856 & 10.1 \\
\hline Nussbaumer et al. (1981) & & & 0.235 & & \\
\hline Quinet et al. (1996) & SST & 0.567 & 0.262 & 0.755 & 6.59 \\
\hline & HFR & 0.500 & 0.220 & 0.694 & 5.20 \\
\hline Rostohar et al. (2001b) & & $0.53(3)$ & $0.23(3)$ & & \\
\hline This work (exp) & & & & $0.65(2)$ & $3.8(3)$ \\
\hline This work (calc) & & & 0.222 & & \\
\hline
\end{tabular}

SST $=$ Superstructure; HFR = Relativistic Hartree-Fock.

that $L S$ coupling is a good approximation for the low-level system in Fe II. Our new calculations of the $a^{6} \mathrm{~S}$ lifetime are also in very good agreement with the measured lifetime.

Quinet et al. (1996) have made calculations using both the relativistic Hartree-Fock (HFR) method in the Cowan code (Cowan 1981) and the Superstructure (SST) code (Eissner et al. 1974; Nussbaumer \& Storey 1978). The SST code gives systematically larger lifetimes values than HFR, but there is no consistency as to which code gives the better value, judged from the experimental values. The values calculated by Garstang (1962) are consistently larger than the experimental values.

The branching fractions derived from the emission line spectrum of gas ejecta in $\eta$ Carinae have been corrected for reddening (see Sect. 3.1). For lines within a limited spectral region this correction is very small. For the other lines separated by about $2500 \AA$ the error introduced is the main contribution to the total uncertainty.

From some of the metastable levels there are a few transitions with significant branching fractions that are not observed in the nebular spectra. Most fall in wavelength regions not covered by the observations. A few are weak transitions from the levels of interest that are too faint to be observed in the stellar spectrum. For all these lines we have used the calculations by Quinet et al. (1996) to estimate the missing branching fractions, the sum of which results in the residual. The residual is $3.5 \%$ for $a^{4} \mathrm{G}_{9 / 2}$ and $0.1 \%$ for $a^{6} \mathrm{~S}_{5 / 2}$.

For $b^{2} \mathrm{H}_{11 / 2}$ we observe only the two transitions down to $a^{4} \mathrm{~F}$. There are two more lines with significant branching ratios, resulting in a residual of $26 \%$. It is interesting to note that the major decay channels $(74 \%)$ for $b^{2} \mathrm{H}_{11 / 2}$ are the transitions to $a^{4} \mathrm{~F}$, the same channels as for $a^{4} \mathrm{G}$. This is a sign of the level mixing between $b^{2} \mathrm{H}$ and $a^{4} \mathrm{G}$, which is further discussed and illustrated in Sect. 6. The lines responsible for the residual BF appear at $9682 \AA$, which is heavily blended, and at $2.03 \mu$, which is outside the observed region. The large residual makes it too uncertain to derive experimental $A$-values for the two transitions observed from $b^{2} \mathrm{H}_{11 / 2}$. However, the calculated branching ratio for these two lines is in good agreement with the observed intensity (see Fig. 3). In Table 6 we give adjusted theoretical $A$-values, i.e. theoretical branching fractions combined with the measured lifetime.

As the forbidden lines from $b^{4} \mathrm{D}_{7 / 2}$ appear in a wider wavelength range, $3100-5600 \AA$, the branching fractions for the transitions depend on the reliability of the reddening curve discussed in Sect. 3.1. However, instead of adopting the calculated $A$-values for these lines we have used the theoretical $A$ values for 14 lines to derive the final branching fractions from the observed intensity ratios in the $\eta$ Carinae spectrum corrected for reddening, as described in Sect. 3. Consequently, the uncertainties of these BF's are larger than for the lines from the three other levels. The calculated residual is $11 \%$ for $b^{4} \mathrm{D}_{7 / 2}$.

For the $A$-values with astrophysical branching ratios and experimental lifetimes we have estimated the uncertainties, which are included in Table 5. The uncertainties of the lifetime and the branching fractions are treated as independent. In the uncertainty of the BF effects from instrument calibration, intensity measurements, calculated residual and reddening are included.

\section{Mixing of the $a^{4} \mathrm{G}_{9 / 2}$ and the $b^{2} \mathrm{H}_{11 / 2}$ levels}

The largest deviation between experimental and theoretical lifetimes occurs for the $b^{2} \mathrm{H}_{11 / 2}$ level, where the experimental value is more than $25 \%$ smaller than the calculated value. Faster decays or more decay channels are thus available for $b^{2} \mathrm{H}_{11 / 2}$ than predicted by theory. The result is a verification of the level mixing between $b^{2} \mathrm{H}_{11 / 2}$ and $a^{4} \mathrm{G}_{11 / 2}$, which has been observed earlier in the study of permitted lines of Fe II (Johansson 1978). The lifetime of the $a^{4} \mathrm{G}_{11 / 2}$ level is predicted to be about 10 times shorter than the lifetime of $b^{2} \mathrm{H}_{11 / 2}$. Unfortunately, due to frequency limitations in the probing laser we have not been able to measure the effect due to mixing on the lifetime of $a^{4} \mathrm{G}_{11 / 2}$.

An astrophysical "proof" for the level mixing is the observation of the $b^{2} \mathrm{H}_{11 / 2}-z^{6} \mathrm{~F}_{9 / 2}$ transition at $6269.97 \AA$ in the solar spectrum, which contradicts all statements about good $L S$ coupling in the lower part of the energy level diagram of Fe II. The transition is a result of level mixing of both the upper and the lower states, i.e. $b^{2} \mathrm{H}_{11 / 2}$ is mixed with $a^{4} \mathrm{G}_{11 / 2}$ and $z^{6} \mathrm{~F}_{9 / 2}$ is mixed with $z^{4} \mathrm{~F}_{9 / 2}$. Thus, the observed line is an appendage to the strong $a^{4} \mathrm{G}_{11 / 2}-z^{4} \mathrm{~F}_{9 / 2}$ transition at $5316.61 \AA$ (Multiplet 49). The line was not identified in the atlas of the solar spectrum by Moore et al. (1966). Since the transition probablilty is very sensitive to a small change in the eigenvector composition of an energy level, there is probably not a need for a large mixing to account for a change in radiative lifetimes that matches the observed deviation from the calculated value. 
Table 5. $A$-values (in s ${ }^{-1}$ ) for $[\mathrm{Fe} \mathrm{II}]$ lines from $a^{4} \mathrm{G}_{9 / 2}, a^{6} \mathrm{~S}_{5 / 2}$ and $b^{4} \mathrm{D}_{7 / 2}$.

\begin{tabular}{|c|c|c|c|c|c|c|c|c|}
\hline \multirow{2}{*}{$\begin{array}{l}\text { Transition } \\
\text { Upper level }\end{array}$} & \multirow[b]{2}{*}{ Lower level } & \multirow[b]{2}{*}{$\lambda(\AA)$} & \multirow{2}{*}{$\begin{array}{l}\text { Experimental } \\
\text { This work }\end{array}$} & \multicolumn{5}{|c|}{ Theoretical } \\
\hline & & & & This work & $Q^{a}(\mathrm{SST})$ & $Q^{b}$ (HFR) & $G^{c}$ & $N^{d}$ \\
\hline \multirow[t]{3}{*}{$a^{4} \mathrm{G}_{9 / 2}$} & $a^{4} \mathrm{~F}_{9 / 2}$ & 4177 & $0.29(0.05)(\mathrm{bl})$ & & 0.184 & 0.194 & 0.14 & \\
\hline & $a^{4} \mathrm{~F}_{7 / 2}$ & 4276 & $0.83(0.07)$ & & 0.750 & 0.819 & 0.65 & \\
\hline & $a^{4} \mathrm{~F}_{5 / 2}$ & 4352 & $0.36(0.04)$ & & 0.345 & 0.380 & 0.31 & \\
\hline \multirow[t]{5}{*}{$a^{6} \mathrm{~S}_{5 / 2}$} & $a^{6} \mathrm{D}_{9 / 2}$ & 4287 & $1.53(0.22)$ & 1.625 & 1.37 & 1.65 & 1.12 & 1.53 \\
\hline & $a^{6} \mathrm{D}_{7 / 2}$ & 4359 & $1.19(0.21)$ & 1.204 & 1.02 & 1.22 & 0.82 & 1.141 \\
\hline & $a^{6} \mathrm{D}_{5 / 2}$ & 4413 & $0.84(0.13)$ & 0.853 & 0.725 & 0.858 & 0.58 & 0.812 \\
\hline & $a^{6} \mathrm{D}_{3 / 2}$ & 4452 & $0.53(0.08)$ & 0.547 & 0.465 & 0.548 & 0.37 & 0.522 \\
\hline & $a^{6} \mathrm{D}_{1 / 2}$ & 4474 & $0.26(0.04)$ & 0.267 & 0.227 & 0.267 & 0.18 & 0.255 \\
\hline \multirow[t]{5}{*}{$b^{4} \mathrm{D}_{7 / 2}$} & $a^{6} \mathrm{D}_{9 / 2}$ & 3175 & $0.23(0.03)$ & & 0.190 & 0.208 & 0.22 & \\
\hline & $a^{4} \mathrm{~F}_{9 / 2}$ & 3376 & $0.96(0.10)$ & & 0.846 & 0.981 & 0.73 & \\
\hline & $a^{4} \mathrm{~F}_{7 / 2}$ & 3440 & $0.23(0.03)$ & & 0.289 & 0.326 & 0.24 & \\
\hline & $a^{4} \mathrm{P}_{5 / 2}$ & 5551 & $0.18(0.04)$ & & 0.149 & 0.176 & 0.13 & \\
\hline & $a^{4} \mathrm{P}_{3 / 2}$ & 5613 & $0.10(0.03)$ & & 0.0808 & 0.0934 & 0.073 & \\
\hline
\end{tabular}

${ }^{a, b}$ Quinet et al. (1996); ${ }^{c}$ Garstang (1962); ${ }^{d}$ Nussbaumer et al. (1981).

Table 6. $A$-values $\left(\mathrm{s}^{-1}\right)$ for transitions from $b^{2} \mathrm{H}_{11 / 2}$. The branching fractions from Quinet et al. (SST, 1996) are combined with the experimental lifetime from this work.

\begin{tabular}{llll}
\hline \hline Transition & & $\lambda(\AA)$ & $A$-value $\left(\mathrm{s}^{-1}\right)$ \\
Upper level & Lower level & & \\
\hline$b^{2} \mathrm{H}_{11 / 2}$ & $a^{4} \mathrm{~F}_{9 / 2}$ & 4114 & 0.14 \\
& $a^{4} \mathrm{~F}_{7 / 2}$ & 4211 & 0.058 \\
& $a^{2} \mathrm{G}_{9 / 2}$ & 9682 & 0.036 \\
\hline
\end{tabular}

\section{Conclusion}

Experimental transition probabilities of 13 lines from three of the four metastable Fe II levels, for which we have measured the radiative lifetimes, have been derived according to Eq. (1). The values are inserted in Table 5 together with values from previous calculations. For the fourth level we give 3 adjusted $A$ values (Table 6). We also give an estimate of the uncertainty of the transition probabilities, see Table 5, which are in the range $10-30 \%$.

Acknowledgements. We are grateful to the staff of the CRYRING facility and appreciate their efforts to provide stable laboratory conditions. This work is supported by the Swedish Research Council (VR). H. Hartman acknowledges travel support from the Swedish Royal Academy of Science (KVA). We also wish to thank PPARC, UK, for support under Grant GR/L20276. The HST/STIS observations were performed under $H S T$ proposal 7302. This research has made use of NASA's Astrophysics Data System Bibliographic Services.

\section{References}

Abrahamsson, K., Andler, G., Bagge, L., et al. 1993, Nucl. Instrum. Methods Phys. Res. B, 79, 269

Bowen, I. S. 1928, ApJ, 67, 1

Brage, T., \& Hibbert, A. 1989, J. Phys. B Atomic Molec. Phys., 22, 713

Clementi, E., \& Roetti, C. 1974, Atomic Data and Nuclear Data Tables, 14, 177
Cowan, R. 1981, The Theory of Atomic Structure and Spectra (University of California Press, Berkeley, California)

Davidson, K., Ebbets, D., Weigelt, G., et al. 1995, AJ, 109, 1784

Donnelly, M. P., \& Hibbert, A. 2001, MNRAS, 321, 247

Eissner, W., Jones, M., \& Nussbaumer, H. 1974, Comput. Phys. Commun., 8, 270

Eriksson, K. K. S. 1965, Ark. Fys., 30, 199

Garstang, R. H. 1962, MNRAS, 124, 321

Gull, T., \& Ishibashi, K. 2001, in Eta Carinae and Other Mysterious Stars: The Hidden Opportunities of Emission Spectroscopy, ed. T. R. Gull, S. Johannson, \& K. Davidson (San Francisco: Astronomical Society of the Pacific), ASP Conf. Proc., 242, 59

Gull, T. R., Ishibashi, K., Davidson, K., \& The Cycle 7 STIS Go Team. 1999, in Eta Carinae at The Millennium, ASP Conf. Ser., 179, 144

Hibbert, A. 1975, Comput. Phys. Commun., 9, 141

Hibbert, A. 1996, Phys. Scr., T65, 104

Hibbert, A., Glass, R., \& Froese Fischer, C. 1991, Comput. Phys. Commun., 64, 455

Johansson, S. 1978, Phys. Scr., 18, 217

Lidberg, J., Al-Khalili, A., Norlin, L.-O., et al. 1999, Nucl. Instrum. Methods Phys. Res. B, 152, 157

Mannervik, S. 2002, Phys. Scr., T100, 81

Mannervik, S., Broström, L., Lidberg, J., Norlin, L., \& Royen, P. 1996, Phys. Rev. Lett., 76, 3675

Merrill, P. W. 1928, ApJ, 67, 391

Moore, C. E., Minnaert, M. G. J., \& Houtgast, J. 1966, The solar spectrum 2935 A to 8770 A (National Bureau of Standards Monograph, Washington: US Government Printing Office (USGPO), 1966)

Nussbaumer, H., Pettini, M., \& Storey, P. J. 1981, A\&A, 102, 351

Nussbaumer, H., \& Storey, P. J. 1978, A\&A, 64, 139

Osterbrock, D. 1989, Astrophysics of Gaseous Nebulae and Active Galactic Nuclei (University Science Books, California)

Quinet, P., Le Dourneuf, M., \& Zeippen, C. J. 1996, A\&AS, 120, 361

Raassen, A., \& Uylings, P. 2000, Atomic data can be found on http://www.science.uva.nl/pub/orth/iron/FeII.E1

Rostohar, D., Andersson, K., Derkatch, A., et al. 2001a, Phys. Scr., 64, 237

Rostohar, D., Derkatch, A., Hartman, H., et al. 2001b, Phys. Rev. Lett., 86,1466

Weigelt, G., \& Ebersberger, J. 1986, A\&A, 163, L5 